SESSION 5

PHYSICS OF THE CRAB NEBULA 


\title{
5.1 PLASMA INTERACTIONS IN THE CRAB NEBULA
}

\author{
F. D. KAHN \\ Astronomy Department, University of Manchester, Manchester, U.K.
}

\begin{abstract}
Alfvén waves can be carried by the thermal plasma in the Crab Nebula. Each such wave perturbs the relativistic plasma present, in particular it strongly affects those particles which are in resonance with it. For a wave travelling parallel to the magnetic field the resonances are quite simple, but a wave travelling obliquely can give rise to multiple resonances, and can therefore couple together particles with quite different energies.

It is shown that the interaction with the relativistic plasma leads to an amplification of the Alfven waves when the mean velocity of relativistic plasma relative to the thermal plasma exceeds the Alfven speed. The rise time for the instability is quite short, and the waves, once excited, are highly effective in redistributing the directions of motion of the relativistic particles. The relativistic plasma therefore cannot stream freely through the thermal plasma. On the other hand the disturbances will never quite die out in the Crab Nebula as long as the Crab pulsar keeps injecting fresh plasma, and thus keeps producing new inhomogeneities in the relativistic plasma density.
\end{abstract}

\section{Introduction}

The Crab Nebula shows unmistakable signs

(i) of containing relativistic charged particles,

(ii) of the presence of a magnetic field,

(iii) of undergoing frequent injections of fresh relativistic particles produced in the Crab pulsar.

The nebula cannot therefore be in a state of equilibrium. Further, even though most of the internal energy of the system is due to relativistic particles and to an (electro) magnetic field, the overall expansion of the nebula takes place at a speed of around $10^{8} \mathrm{~cm} \mathrm{sec}^{-1}$, much less than the speed of light. Since the gravitational potential within the nebula is negligibly small, it follows that the inertia of the thermal plasma present holds back the relativistic plasma and stops it from getting away much faster. It therefore becomes important to study interactions between relativistic and thermal plasmas.

I shall discuss one way in which such an interaction can arise, through a coupling via the Alfvén waves carried by the thermal plasma. The properties of Alfvén waves are easily derived. The perfect conductivity condition requires that a plasma always responds to low frequency fluctuations in such a way that there is no electric field with respect to the rest frame of the plasma, or that

$$
\mathbf{E}+\frac{\mathbf{u}}{c} \wedge \mathbf{H}_{0}=0
$$

where $\mathbf{u}$ is plasma velocity, $\mathbf{H}_{0} \equiv\left(0,0, H_{0}\right)$ is the zero-order magnetic field, and $\mathbf{E}$ is the electric field. 
Note that $\mathbf{E}$ is perpendicular to $\mathbf{H}_{0}$. The vector potential $\mathbf{A}$ of the Alfvén wave may be defined by

$$
\mathbf{E}=-\frac{1}{c} \frac{\partial \mathbf{A}}{\partial t}
$$

expressed in components

$$
\mathbf{A}=\left(A_{1}, A_{2}, 0\right)
$$

If the wave has a space-time dependence of the form exp $i\left(k_{1} x_{1}+k_{3} x_{3}-\omega t\right)$, it follows from (1), (2) and (3) that

$$
i \omega\left(A_{1}, A_{2}, 0\right)+H_{0}\left(u_{2},-u_{1}, 0\right)=0 .
$$

The linearized equation of motion for the plasma is

$$
\varrho_{0} \frac{\partial \mathbf{u}}{\partial t}=\frac{1}{c} \mathbf{j} \wedge \mathbf{H}_{0}
$$

where $\varrho_{0}$ is the density of the thermal plasma, and where the current density is denoted by

$$
\mathbf{j}=\frac{c}{4 \pi} \nabla \wedge(\nabla \wedge \mathbf{A})=\frac{c}{4 \pi}\left\{k_{3}^{2} A_{1},\left(k_{1}^{2}+k_{3}^{2}\right) A_{2},-k_{1} k_{3} A_{1}\right\}
$$

Equation (4) now becomes

$$
-i \omega \varrho_{0}\left(u_{1}, u_{2}, u_{3}\right)=\frac{H_{0}}{4 \pi}\left\{\left(k_{1}^{2}+k_{3}^{2}\right) A_{2},-k_{3}^{2} A_{1}, 0\right\} .
$$

Thus $u_{3} \equiv 0$, and the wave motion splits into two modes. One mode is described by Mode I:

and

$$
i \omega A_{1}+u_{2} H_{0}=0
$$

$$
i \omega \varrho_{0} u_{2}-\frac{k_{3}^{2} H_{0}}{4 \pi} A_{1}=0 \text {. }
$$

The waves of this mode obey the dispersion relation

$$
\omega^{2}=\frac{H_{0}^{2}}{4 \pi \varrho_{0}} k_{3}^{2} \equiv v_{A}^{2} k_{3}^{2},
$$

and the components of the group velocity are

$$
\partial \omega / \partial k_{1}=0, \quad \partial \omega / \partial k_{3}= \pm v_{A} .
$$

Energy therefore propagates parallel to the three-axis with speed $\pm v_{A}$. The space-time dependence of the wave has the form

$$
\exp i\left\{k_{1} x_{1}+k_{3}\left(x_{3}-v_{A} t\right)\right\} \text {. }
$$


The other mode of the wave is described by

Mode II:

$$
i \omega A_{2}-u_{1} H_{0}=0
$$

and

$$
i \omega \varrho_{0} u_{1}+\frac{1}{4 \pi}\left(k_{1}^{2}+k_{3}^{2}\right) H_{0} A_{2}=0,
$$

with the dispersion relation

$$
\omega^{2}=v_{A}^{2}\left(k_{1}^{2}+k_{3}^{2}\right) \text {. }
$$

The group velocity now has components

$$
\partial \omega / \partial k_{1}=v_{A} \sin \alpha, \quad \partial \omega / \partial k_{3}=v_{A} \cos \alpha,
$$

where the wave-vector $\mathbf{k}$ makes an angle $\alpha \equiv \tan ^{-1} k_{1} / k_{3}$ with the three-axis. The space-time dependence of the waves in this mode is

$$
\exp i\left\{\left(k_{1} x_{1}+k_{3} x_{3}\right)-\left(k_{1}^{2}+k_{3}^{2}\right)^{1 / 2} v_{A} t\right\} \text {. }
$$

The Alfvén waves are thus split into two linearly polarized modes, with different frequencies. Degeneracy occurs only if $k_{1}\left(=k_{2}\right)=0$. In that case it is possible to describe the waves in terms of independent, circularly polarized modes.

\section{Response of the Relativistic Plasma to Alfuén Waves}

I shall now consider how the relativistic plasma is affected by the Alfvén waves, and later find a condition under which the resulting interaction leads to an instability. For the sake of simplicity the wave-vector $\mathbf{k}$ of the Alfvén wave is taken to be parallel to the three-axis. In that case one can assume that the wave is circularly polarized, and has a vector potential given by

$$
\mathbf{A}=A(1, i, 0) \exp \left\{i k_{3}\left(x_{3}-v_{A} t\right)\right\} .
$$

To illustrate a simple case, let the relativistic plasma present have a momentum distribution which is isotropic with respect to a frame of reference $\Sigma_{R}$, where $\Sigma_{R}$ moves with a velocity $\left(0,0, v_{R}\right)$ relative to the rest-frame $\Sigma$ of the thermal plasma. With respect to $\Sigma_{R}$, the momentum distribution is

$$
f_{R}=F_{0}\left(p_{\|, R}^{2}+p_{\perp}^{2}\right)
$$

where $p_{\|, R}, p_{\perp}$ are the components of momentum parallel and perpendicular to the three-axis as seen from $\Sigma_{R}$. Relative to the frame of reference $\Sigma$, the distribution function becomes

$$
\begin{aligned}
f_{0} & =F_{0}\left(p_{\|}^{2}+p_{\perp}^{2}-\frac{2 v_{R} \mathscr{E}}{c^{2}} p_{\|}\right) \\
& =F_{0}\left(p_{\|}^{2}+p_{\perp}^{2}\right)-\frac{2 v_{R} \mathscr{E}}{c^{2}} p_{\|} F_{0}^{\prime}\left(p_{\|}^{2}+p_{\perp}^{2}\right)
\end{aligned}
$$


This result is correct to order $v_{R} / c$, and therefore good enough, unless the relative mean velocity of the two plasmas is very large. In Equation (19), $p_{\|}$is the threecomponent of momentum and $\mathscr{E}$ the energy of a particle, with respect to $\Sigma$. The linearized Vlasov equation for the relativistic plasma is

\section{I define}

$$
\frac{\partial f}{\partial t}+\dot{x}_{3} \frac{\partial f}{\partial x_{3}}+\dot{\varphi} \frac{\partial f}{\partial \varphi}+\dot{p}_{\|} \frac{\partial f}{\partial p_{\|}}+\dot{p}_{\perp} \frac{\partial f}{\partial p_{\perp}}=0
$$

$$
\varphi=\tan ^{-1} p_{2} / p_{1}
$$

where $p_{1}, p_{2}$ are the one and two components of momentum.

Equation (20) is to be linearized and then solved. For this purpose note that $\partial f / \partial x_{3}$ and $\partial f / \partial \varphi$ both vanish in the undisturbed plasma, which is spatially homogeneous, and has axial symmetry in momentum space with respect to the three-axis. The coefficients of these two terms need only be calculated to zero order, since $\partial f / \partial x_{3}$ and $\partial f / \partial \varphi$ can only appear as first order quantities. The coefficients are $\dot{x}_{3}=c^{2} p_{\|} / \mathscr{E}$, and $\dot{\varphi}=-e c H_{0} / \mathscr{E}$; the first of these results is obvious from the relation of velocity and momentum, and the second describes the Larmor precession of a charged particle in the undisturbed magnetic field.

On the other hand, both $p_{\|}$and $p_{\perp}$ stay constant in the undisturbed field, and therefore $\dot{p}_{\|}$and $\dot{p}_{\perp}$ are first order quantities. It is then enough to insert for $\partial f / \partial p_{\|}$ and $\partial f / \partial p_{\perp}$ the zero-order quantities $\partial f_{0} / \partial p_{\|}$and $\partial f_{0} / \partial p_{\perp}$.

The rates of change of $p_{\|}$and $p_{\perp}$ can be found in terms of the vector potential $\mathbf{A}$. A brief calculation yields that

$$
\left.\begin{array}{l}
\dot{p}_{\|}=\frac{i k_{3} e A c}{\mathscr{E}} p_{\perp} e^{i \varphi} \\
\dot{p}_{\perp}=\frac{i k_{3} e A}{c}\left(v_{A}-\frac{c^{2} p_{\|}}{\mathscr{E}}\right) e^{i \varphi} .
\end{array}\right\}
$$

When these quantities are substituted into the linearized equation, and use is made of the assumed space-time dependence (17) of the wave, it turns out that, correct to the first order, the change in the plasma distribution function is given by

$$
f^{(1)}\left\{\frac{k_{3} c^{2} p_{\|}}{\mathscr{E}}-k v_{A}-\frac{c e H_{0}}{\mathscr{E}}\right\}=\frac{k_{3} e A c}{\mathscr{E}}\left\{\left(p_{\|}-\frac{\mathscr{E} v_{A}}{c^{2}}\right) \frac{\partial f_{0}}{\partial p_{\perp}}-p_{\perp} \frac{\partial f_{0}}{\partial p_{\|}}\right\}
$$

I now substitute for $f_{0}$ from equation (19) and find that

$$
f^{(1)}=\frac{\left(2 \mathscr{E} p_{\perp} / c^{2}\right)(e A / c)\left(v_{R}-v_{A}\right) F_{0}^{\prime}}{p_{\|}-\mathscr{E} v_{A} / c^{2}-e H_{0} / c k_{3}}
$$

$f^{(1)}$ becomes large for particles that resonate with the wave, that is for particles which satisfy

$$
p_{\|}=\frac{\mathscr{E} v_{A}}{c^{2}}+\frac{e H_{0}}{c k_{3}}
$$


The resonance depends, essentially, on the $p_{\|}, k_{3}$ relation. It also depends on the sense of polarization of the wave. The present calculation is done in terms of a circularly polarized wave: to reverse the sense of polarization we need only change $k_{3}$ to $-k_{3}$. However, a plane polarized wave can be split into two circularly polarized waves with opposite senses of polarization, and it will therefore resonate with particles at two different values of $p_{\|}$.

The resonance condition (25) has a simple interpretation. The components of $\mathbf{p}_{\perp}$ for a particle in its zero order trajectory are

$$
\left(p_{1}, p_{2}\right)=p_{\perp}\left\{\cos \left(\frac{c e H_{0}}{\mathscr{E}} t+\varepsilon\right),-\sin \left(\frac{c e H_{0}}{\mathscr{E}} t+\varepsilon\right)\right\},
$$

where $\varepsilon$ is a phase factor. The one and two components of the vector potential are

$$
\begin{aligned}
\left(A_{1}, A_{2}\right) & =A\left\{\cos \left(k_{3} x_{3}-\omega t\right), \sin \left(k_{3} x_{3}-\omega t\right)\right\} \\
& =A\left\{\cos \left(\frac{k_{3} c^{2} p_{\|}}{\mathscr{E}}-\omega\right) t, \sin \left(\frac{k_{3} c^{2} p_{\|}}{\mathscr{E}}-\omega\right) t\right\}
\end{aligned}
$$

at the position of the particle in its zero order orbit. Resonance occurs when the scalar product $\mathbf{p}_{\perp}$. A has a phase factor which is independent of time, that is when

$$
-\frac{c e H_{0}}{\mathscr{E}}=\frac{k_{3} c^{2} p_{\|}}{\mathscr{E}}-\omega
$$

With $\omega$ set equal to $k_{3} v_{A}$, conditions (25) and (28) are found to be equivalent.

\section{Alfvén Waves which Propagate Obliquely}

The interaction of the relativistic plasma with an Alfvén wave becomes somewhat more complex when the wave-vector $\mathbf{k}$ is not parallel to the magnetic field. As was mentioned before, the Alfvén wave now has two independent plane polarized modes, I and II. Each of these modes can be analysed into two circularly polarized waves, with opposite senses of polarization, but the component waves are now no longer independent, and must always be considered together.

Another important modification occurs in the resonance condition. The components of $\mathbf{p}_{\perp}$ are, once again

$$
\left(p_{1}, p_{2}\right)=p_{\perp}\left\{\cos \left(\frac{c e H_{0}}{\mathscr{E}} t+\varepsilon\right),-\sin \left(\frac{c e H_{0}}{\mathscr{E}} t+\varepsilon\right)\right\},
$$

for the zero order particle trajectory; the components of the vector potential become

$$
\left(A_{1}, A_{2}\right)=A\left\{\cos \left(k_{1} x_{1}+k_{3} x_{3}-\omega t\right), \sin \left(k_{1} x_{1}+k_{3} x_{3}-\omega t\right)\right\} .
$$

The same substitution as before can be made for $x_{3}$, and for the other position variable it is found that

$$
x_{1}=\int v_{1} \mathrm{~d} t=c_{\mathscr{E}}^{c^{2}} \int p_{1} \mathrm{~d} t={ }_{e H_{0}}^{c p_{\perp}} \sin \left(\frac{c e H_{0}}{\mathscr{E}} t+\varepsilon\right) .
$$


In the complex notation the phase dependence of the vector potential at the position of the particle can now be expressed by

$$
\mathscr{A} \equiv A_{1}+i A_{2}=A \exp i\left\{\frac{k_{1} c p_{\perp}}{e H_{0}} \sin \left(\frac{c e H_{0}}{\mathscr{E}} t+\varepsilon\right)+\left(\frac{k_{3} c^{2} p_{\|}}{\mathscr{E}}-\omega\right) t\right\}
$$

the corresponding relation for $\mathbf{p}_{\perp}$ is

$$
\mathscr{P} \equiv p_{1}+i p_{2}=p_{\perp} \exp \left\{-i\left(\frac{c e H_{0}}{\mathscr{E}} t+\varepsilon\right)\right\} .
$$

The presence of the sine term in the exponent in (32) somewhat changes the phase relation. On using the series expansion

$$
e^{i z \sin \theta}=\sum_{-\infty}^{\infty} J_{r}(z) e^{i r \theta}
$$

relation (32) may be re-written in the form

$$
\begin{aligned}
\mathscr{A} & =A \sum_{-\infty}^{\infty} J_{r}\left(\frac{k_{1} c p_{\perp}}{e H_{0}}\right) \exp i r\left(\frac{c e H_{0}}{\mathscr{E}} t+\varepsilon\right) \exp i\left(\frac{k_{3} c^{2} p_{\|}}{\mathscr{E}}-\omega\right) t \\
& \equiv \sum_{-\infty}^{\infty} \mathscr{A}_{r} \exp \operatorname{ir}\left(\frac{c e H_{0}}{\mathscr{E}} t+\varepsilon\right) \exp i\left(\frac{k_{3} c^{2} p_{\|}}{\mathscr{E}}-\omega\right) t .
\end{aligned}
$$

The particle can now have a resonance with any one of the infinite number of components into which the expression for $\mathscr{A}$ has been analysed. The condition for resonance with the $r$ th component is clearly

$$
\frac{c e H_{0}}{\mathscr{E}}=r \frac{c e H_{0}}{\mathscr{E}}+k_{3} \frac{c^{2} p_{\|}}{\mathscr{E}}-\omega
$$

or

$$
p_{\|}=\frac{\mathscr{E} \omega}{c^{2} k_{3}}-(r-1) \frac{e H_{0}}{c k_{3}}
$$

A given wave can now resonate with a much larger number of particles. The importance of the $r$ th resonance is indicated by the value of the coupling coefficient $J_{r}\left(k_{1} c p_{\perp} / e H_{0}\right)$. If $k_{1}=0$, then all these coefficients are zero, except that for $r=0$. In that case condition (36) is identical with condition (25) (when $\omega / k$ is set equal to $v_{A}$ ).

However, interesting new effects can arise when there are high resonances, for which $r \gg 1$. The coupling coefficient $J_{r}\left(k_{1} c p_{\perp} / e H_{0}\right)$ for such a resonance is appreciable only when

$$
r \approx k_{1} c p_{\perp} / e H_{0}
$$

that is when the argument of the Bessel function is approximately equal to its order. I shall now compare relation (37) with relation (36), but shall first simplify the latter 
by noting that the term $\mathscr{E} \omega / c^{2} k_{3}$ is small compared with the momentum of the particle, since the phase velocity of the Alfven wave will certainly be small compared with the speed of light. Relation (36) is therefore, to a good enough approximation,

$$
p_{\|} \doteqdot-(r-1){ }_{c k_{3}}^{e H_{0}} \doteqdot-r{ }_{c k_{3}}^{e H_{0}} .
$$

Two results now follow: first the wave-number $k$ of the Alfvén wave is related to the momentum of the resonating particle by

$$
k \doteqdot \stackrel{r e H_{0}}{c p}
$$

In a high resonance a particle of given energy resonates with a wave having a much larger wave-number (and much smaller wavelength) than that of the wave with which the particle is in zero resonance. However, the resonant wave-number varies inversely as the momentum (or the energy) of the relativistic particle. It therefore is possible for a given wave to resonate at the same time with a particle of low energy, in a low resonance, and a particle of high energy, in a high resonance. By this means the high energy particles can be coupled to the low energy particles in the relativistic plasma, or, more generally, the whole range of energies can be coupled together.

It also follows from equations (37) and (38) that

$$
p_{\perp} / p_{\|} \doteqdot-k_{3} / k_{1}=-\cot \alpha \text {. }
$$

High resonance therefore always occurs with particles which are travelling nearly at right angles to the wave-vector $\mathbf{k}$, or almost in planes of constant phase of the Alfvén wave.

\section{The Growth Rate for Unstable Waves}

The full treatment of the properties of oblique waves will clearly be rather complex. To illustrate the argument I therefore return, now, to Alfvén waves for which $k_{1}=0$, that is to waves whose wave-vector is parallel to the magnetic field. The disturbance caused by the wave sets up a current in the relativistic plasma given by

$$
\mathbf{j}=N_{R} e \int_{0}^{\infty} \int_{-\infty}^{\infty} \int_{0}^{2 \pi} \frac{c^{2}}{\mathscr{E}}\left(p_{\perp} \cos \varphi, p_{\perp} \sin \varphi, p_{\|}\right) f^{(1)} p_{\perp} \mathrm{d} p_{\perp} \mathrm{d} p_{\|} \mathrm{d} \varphi
$$

In this formula $N_{R}$ is the number of particles per $\mathrm{cm}^{3}$ in the relativistic plasma, and the form of $f^{(1)}$ is given by relation (24) in terms of the perturbing wave and the zero order particle distribution. The zero order distribution is assumed to be normalized to unity, and the phase factor $\exp i\left\{k_{3}\left(x_{3}-v_{A} t\right)\right\}$ is assumed to be understood. The integration past the singularity of $f^{(1)}$, at $p_{\|}=\left(\mathscr{E} v_{A} / c^{2}\right)+\left(e H_{0} / c k_{3}\right)$, is carried out in the usual manner by deforming the contour in such a way that the calculation gives 
the value obtained when the frequency $\omega$ tends to the real axis from the upper half of the complex- $\omega$ plane. The result is that

$$
\mathbf{j}=-i\left(\operatorname{sgn} k_{3}\right) \frac{2 \pi^{2} N_{R} e^{2}}{c} A\left(v_{R}-v_{A}\right)(1, i, 0) \int_{0}^{\infty} p_{\perp} F_{0}\left(p_{\|}^{* 2}+p_{\perp}^{2}\right) \mathrm{d} p_{\|}
$$

after some manipulation. $p_{\|}^{*}$ is the resonant value of $p_{\|}$. For comparison the electric field of the Alfvén wave is

$$
\mathbf{E}=\frac{i k_{3} v_{A}}{c} A(1, i, 0)
$$

and $\mathbf{E} \cdot \mathbf{j}$ is negative when

$$
\left|k_{3}\right| v_{A}\left(v_{R}-v_{A}\right)>0 \text {. }
$$

In that case the plasma response is such that work is done by the plasma on the wave. In other words the wave draws energy from the plasma, and grows. The condition for instability can be written

$$
\left|v_{R}\right|>\left|v_{A}\right|
$$

and this means that instability will occur whenever the diffusion speed $v_{R}$ of the relativistic plasma, with respect to the thermal plasma, exceeds the Alfvén speed $v_{\boldsymbol{A}}$. The growth rate $\sigma$ of the instability is given by

$$
\sigma=\frac{1}{2} \frac{\text { rate at which work is done on the wave } / \mathrm{cm}^{3}}{\text { energy density of the wave }}
$$

I make the estimate that a typical value of

$$
\int_{0}^{\infty} p_{\perp} F_{0}\left(p_{\|}^{* 2}+p_{\perp}^{2}\right) \mathrm{d} p_{\perp}
$$

is of order

$$
\frac{1}{2 \pi p_{\|}^{*}} \sim \frac{c}{2 \pi \widetilde{E}}
$$

where $\overline{\mathscr{E}}$ is a typical energy in the plasma. Further, from relation (25)

$$
p_{\|} \sim \overline{\mathscr{E}} / c \sim e H_{0} / c k_{3},
$$

and now substituting into equation (46) I find that, by order of magnitude,

$$
\sigma \sim \frac{4 \pi^{2} N_{R} e v_{A}\left(v_{R}-v_{A}\right)}{c H_{0}} \sim \frac{\pi N_{R} e H_{0}}{c \varrho_{0}}
$$

if $v_{R}$ is of the order of $v_{A}\left(\equiv \sqrt{H_{0}^{2} / 4 \pi \varrho_{0}}\right)$. 
Typical values for the Crab Nebula can be substituted into (47) by setting, for example,

$$
\begin{aligned}
N_{R} & =\text { number density of relativistic particles }=10^{-6} \mathrm{~cm}^{-3}, \\
H_{0} & =10^{-4} \mathrm{G} \\
\varrho_{0} & =\text { thermal plasma density }=10^{-21} \mathrm{~g} / \mathrm{cm}^{3},
\end{aligned}
$$

with the result that

$$
\sigma \approx 5 \times 10^{-9} \mathrm{sec}^{-1} \equiv 0.17 \mathrm{yr}^{-1}
$$

The growth rate can therefore be quite large. It is even larger for larger assumed values of the relative diffusion speed $v_{R}$.

\section{The Redistribution of Particle Momenta by the Wave}

It remains to estimate how rapidly the particle momenta are redistributed by the ensemble of Alfvén waves which result from the instability. The major effect leading to the redistribution is the acceleration of the charged particles by the disturbance magnetic field $H^{(1)}$. There is also an acceleration due to the disturbance electric field $E^{(1)}$. But this field is of order $\left(v_{A} / c\right)$ times $H^{(1)}$, and therefore small enough to be neglected here.

Consider now a typical particle whose energy is $\mathscr{E}$ and momentum $p=\mathscr{E} / c$. It resonates with the Alfvén wave whose wavenumber is $k_{3} \sim e H_{0} / \mathscr{E} \equiv k^{*}$, say. A wave with a nearby wave-number, $k^{*}+\delta k$, will change its phase relative to the resonant wave at a rate such that the change in phase as observed at the moving particle is

$$
\begin{aligned}
\delta \varphi & =\left(v_{\|}-v_{A}\right) T \delta k \\
& \doteqdot v_{\|} T \delta k
\end{aligned}
$$

after time $T$. During the time interval $T$ the waves which lie within a range

$$
\delta k \approx 1 / v_{\|} T
$$

around $k^{*}$ will therefore contribute coherently to the magnetic field deflecting the particle. If the Fourier decomposition of the magnetic field is given by

$$
H=\int \mathscr{H}(k) e^{i k\left(x_{3}-v_{A} t\right)} \mathrm{d} k
$$

then the effective magnetic field $H_{\text {eff }}$ which acts on the particle during the interval $T$ is

$$
H_{\text {eff }}=\int_{\delta k} \mathscr{H}(k) e^{i k\left(x_{3}-v_{A} t\right)} \mathrm{d} k .
$$

In a random field there are no correlations between contributions from different wavenumbers. Hence the expectation value of the effective magnetic field is

$$
\left\langle H_{\text {eff }}\right\rangle=0
$$


and the mean square effective field is

$$
\left\langle H_{\text {eff }}^{2}\right\rangle=\left|\mathscr{H}^{2}\left(k^{*}\right)\right| \delta k
$$

The mean square deflection of a particle is therefore given by

$$
\begin{aligned}
\left\langle\delta \theta^{2}\right\rangle & =\frac{c^{2} e^{2}\left\langle H_{\mathrm{eff}}^{2}\right\rangle}{\mathscr{E}^{2}} T^{2} \\
& =\frac{c^{2} e^{2}\left|\mathscr{H}^{2}\left(k^{*}\right)\right|}{\mathscr{E}^{2} v_{\|}} T .
\end{aligned}
$$

The characteristic time during which the particle momenta are redistributed is given by the setting $\left\langle\delta \theta^{2}\right\rangle$ equal to unity, and becomes

with $v_{\|} \approx c$.

$$
T_{\text {redist. }}=\frac{\mathscr{E}^{2} v_{\|}}{c^{2} e^{2}\left|\mathscr{H}^{2}\left(k^{*}\right)\right|} \approx \frac{\mathscr{E}^{2}}{c e^{2}\left|\mathscr{H}^{2}\left(k^{*}\right)\right|}
$$

The mean free path for a relativistic particle is therefore of the order of

$$
l=c T_{\text {redist. }} \approx \frac{\mathscr{E}^{2}}{e^{2}\left|\mathscr{H}^{2}\left(k^{*}\right)\right|} .
$$

If the spectrum of the Alfvén waves were known it would now be possible to calculate mean free paths for particles of various energies. However, it does not seem worth doing this in detail until the various possible interactions between the waves and the particles have been fully studied. I shall only make an order of magnitude estimate. It follows from the relation

$$
k^{*} \approx e H_{0} / \mathscr{E}
$$

that the width of the Alfvén wave spectrum $\Delta k$ is related to the typical particle energy $\overline{\mathscr{E}}$ by

$$
\Delta k \approx e H_{0} / \overline{\mathscr{E}}
$$

If $\overline{H_{w}^{2}}$ is the mean square field due to the Alfvén waves, then

$$
l \sim \frac{\mathscr{E}^{2} \Delta k}{e^{2}\left|\mathscr{H}^{2}\left(k^{*}\right)\right| \Delta k} \sim \frac{\overline{\mathscr{E}} e H_{0}}{e^{2} \bar{H}_{w}^{2}}=\frac{\overline{\mathscr{E}} H_{0}}{e \bar{H}_{w}^{2}} .
$$

If further the rms wave field $\sqrt{H_{w}^{2}}$ is, say, $\beta$ times the undisturbed field $H_{0}$, then

$$
l \sim \overline{\mathscr{E}} / \beta e H_{0} \sim 2 \times 10^{10} \beta^{-1} \mathrm{~cm}
$$

in the Crab Nebula. Quite moderate amplitudes of the Alfvén waves can thus result in very short mean free paths for the particles in the relativistic plasma. 


\section{Conclusions}

It is very likely that Alfvén waves are readily excited and play an important role in the physics of the Crab Nebula. They lead to the following effects:

(i) The relativistic plasma can only diffuse slowly with respect to the thermal plasma. The pressure in the relativistic plasma therefore acts on the thermal plasma, and governs its motion.

(ii) The Alfven waves redistribute the directions of motion of the particles in the relativistic plasma. The mean free path for a relativistic particle is quite short, and the wave particle interaction is therefore quite effective in making momentum distributions more isotropic.

(iii) However, since the mean free path for a plasma particle is finite, there can never be complete isotropy while there are inhomogeneities in the plasma density. In particular some diffusion of the relativistic plasma will occur, relative to the thermal plasma. As long as the Crab pulsar keeps injecting new plasma, inhomogeneities will continue to be produced and Alfvén waves will be generated.

(iv) Equation (54) suggests that particles with a large energy $\mathscr{E}$ will have a longer mean free path than particles with small energy. If so, the degree of anisotropy should be larger among the high energy particles than among the low energy particles of the relativistic plasma. This suggests that the particles with a large value of $\mathscr{E}$ in the plasma could still be unstable in places where the particles with low $\mathscr{E}$ are stable. As a result energy might possibly be transferred via an Alfvén wave, with which the high- $\mathscr{E}$ particles have a high resonance and the low- $\mathscr{E}$ particles a low resonance. In this way additional energy could be supplied to the electrons, and this may extend the time during which they can emit synchrotron radiation.

\section{References}

Details of the physics of the Crab Nebula are given in several other papers contributed to this symposium.

The theory of Alfvén waves and of plasma waves has been discussed by many authors, including

P. C. Clemmow and J. P. Dougherty, Electrodynamics of Particles and Plasmas, Addison Wesley, 1969.

The importance of the interaction of relativistic plasmas and Alfven waves in the interstellar plasma has been emphasied by a number of authors. The effect is essentially the same as that in the Crab Nebula. References are

R. M. Kulsrud and W. P. Pearce: 1969, Astrophys. J. 156, 445.

J. Skilling: 1970, Monthly Notices Roy. Astron. Soc. 147, 1.

D. G. Wentzel: 1969, Astrophys. J. 156, 303.

Skilling in particular gives an interesting discussion of the associated physical effects.

\section{Discussion}

$J . P$. Ostriker: Your mechanism is similar to the one proposed to stop the streaming of cosmic rays. F. D. Kahn: Yes - it is a standard result. 